\title{
Phase Separation Process in Polymer Systems. II. Microscopic Studies on a Polystyrene and Diisodecyl Phthalate Mixture
}

\author{
Shuichi NoJima, Kenji SHIroshitA, and Takuhei NosE \\ Department of Polymer Chemistry, Tokyo Institute of Technology, \\ Ookayama, Meguro-ku, Tokyo 152, Japan.
}

(Received October 30, 1981)

\begin{abstract}
Late stages of phase separation in liquid mixtures of polystyrene (PS) and diisodecyl phthalate were investigated by microscopic observation. Droplet structures were observed in the matrix. Average droplet diameters $\bar{d}$ for mixtures of 5.7 and $16.6 \mathrm{wt} \%$ PS varied with the period of phase separation $t$ according to a power-law, $\bar{d} \propto t^{\phi}$, with $\phi=0.30( \pm 0.09)$ being independent of quench depth. This value of $\phi$ agreed well with $1 / 3$, theoretically predicted on the basis of the coalescence mechanism and the Ostwald Ripening mechanism. For a blend of PS and poly(methylphenylsiloxane), we investigated the relation between the average droplet diameter and the wave length at the maximum intensity of scattered light observed in the early stage.

KEY WORDS Phase Separation /Late Stage / Droplet Structure / Coarsening / Coalescence /Ostwald Ripening /
\end{abstract}

There are two different mechanisms for the coarsening of droplet phases in the late stage of phase separation ${ }^{1}$ : coalescence and Ostwald Ripening (OR). By the coalescence mechanism, droplets of the daughter phase impinge with each other by translational diffusion and are united into a new droplet. Time dependence of average droplet size $l$ at separation temperature $T$ is theoretically predicted as ${ }^{1}$

$$
l \propto c\left(k_{\mathrm{B}} T \psi / \eta\right)^{1 / 3} t^{1 / 3}
$$

where $\eta$ is the viscosity of the matrix, $\psi$ is the total volume fraction of droplets, and $k_{\mathrm{B}}$ is Boltzmann's constant. By the OR mechanism, large droplets become bigger and small droplets become even more diminished in size with time by evaporation and condensation of the components on droplet surfaces. Theoretical treatment of the OR mechanism was first made by Lifshitz and Slyozov ${ }^{2}$ and by Wagner ${ }^{3}$ for supersaturated solid solutions. For a liquid-liquid phase separation, $l$ is expressed by

$$
l \propto\left[\sigma M /\left(c_{\alpha}-c_{\beta}\right)^{2}\right]^{1 / 3} t^{1 / 3}
$$

where $M$ is the mobility defined phenomenologically, $\sigma$ is the interfacial tension, and $c_{\alpha}$ and $c_{\beta}$ are the equilibrium concentrations of two phases coexisting at the separation temperature $T$. Thus, the same power-law relation $l \propto t^{\phi}$ with $\phi=1 / 3$ holds both for the coalescence and OR mechanisms.

A few observations have been made for the late stages of phase separation in binary mixtures of small molecules ${ }^{4}$ and polymer blends. ${ }^{5-7}$ Goldburg et al. studied a critical mixture of 2-6 lutidine and water by a microscope and found that the droplets grew in accordance with the power-law with an exponent $\phi=1.7$, which is much larger than the theoretical value, $\phi=1 / 3$. This discrepancy can be explained by the effect of gravity on the coarsening of the dispersed phases. McMaster ${ }^{6}$ investigated the coarsening process in mixtures of poly(methyl methacrylate) and styrene-acrylonitrile copolymer, and observed a significant difference in coarsening rate between the dispersed-phase structures and interconnected-phase structures; the former coarsened more slowly than the latter. It was concluded that the dispersed-phases coarsened by the OR mechanism.

The present paper reports a microscopic study on 
the coarsening process in liquid mixtures of polystyrene (PS) and diisodecyl phthalate (DIDP) and those of PS and poly(methylphenylsiloxane) (PMPS). The former system was chosen for the following two reasons. First, the rate of phase separation is appropriate for observing the late stage of phase separation. Second, the refractive indices of pure components are very different from each other, and this condition is favorable for microscopic observation. The latter system was used to relate the average droplet diameter $\bar{d}$ to the wave length ${ }^{8}$ at the maximum intensity of scattered light at the early stage of phase separation.

\section{EXPERIMENTAL}

\section{Materials}

The PS sample used for the PS-DIDP system was a product of Pressure Chem. Co. Its weight-average molecular weight $M_{w}$ was 110,000 and the ratio of $M_{w}$ to the number-average molecular weight $M_{n}$ was less than 1.06. The DIDP sample was a commercial product of Tokyo Chem. Co. and dried under vacuum for $10 \mathrm{~h}$ at $90^{\circ} \mathrm{C}$ before use. The PS sample for the PS-PMPS system was also a product of Pressure Chem. Co. and its $M_{w}$ and $M_{w} / M_{n}$ were 9,000 and less than 1.06 , respectively. The PMPS sample was a product of Toray Silicone Co. and its $M_{w}$ and $M_{w} / M_{n}$ determined by gel permeation chromatography were 2,800 and 1.57 , respectively.

\section{Determination of Cloud Points and Spinodal Points}

We obtained cloud point temperatures $T_{\mathrm{c}}(0)$ by the following method. First, cloud points $T_{\mathrm{c}}(R)$ were measured as a function of cooling rate $R$ from the decrease in intensity of light transmitted through a sample cell with an optical path of $1 \mathrm{~mm}$. Plots of $T_{\mathrm{c}}(R)$ vs. $R$ showed an approximately straight line at any concentration. This allowed us to extrapolate $T_{\mathrm{c}}(R)$ with $R=0$ to obtain $T_{\mathrm{c}}(0)$. The slope of the straight line was largely dependent on the concentration for mixtures of PS and PMPS, ${ }^{9}$ but nearly constant for the PS and DIDP mixtures.

Spinodal temperatures $T_{\mathrm{s}}$ for the mixtures of PS and DIDP were obtained by light scattering measurements by the photon counting method. The details of this apparatus are presented elsewhere. ${ }^{10}$ It was assumed that the scattered intensity $I(0)$ extrapolated to the zero scattering angle at a temperature near $T_{\mathrm{s}}$ could be expressed by

$$
I(0) \propto\left(T-T_{\mathrm{s}}\right)^{-\gamma}
$$

with $\gamma=1.26 .^{11}$ First, we obtained the angular dependence of the scattered intensity $I(\theta)$ at a fixed temperature $T$, and then evaluated $I(0)$ as a function of $T$ by extrapolating the plot of $I(\theta) v s \cdot \sin ^{2}(\theta / 2)$ to $\theta=0$; the plot was linear. Next, $T_{\mathrm{s}}$ was determined from a point at which $I(0)^{-1 / \gamma}$ became zero in the plot of $I(0)^{-1 / \gamma} v s . T$.

\section{Microscopic Observation}

The separation process was followed with an optical microscope with the magnification of 100 . Average droplet diameters $\bar{d}$ at various temperatures and times of phase separation were obtained from a photographic analysis. Phase separation was allowed to occur by the temperature quenching method. The quenching was carried out by moving the sample cell of $50 \mu \mathrm{m}$ in thickness rapidly from an external thermostat controlled just above the cloud point $T_{\mathrm{c}}(0)$ into a hot stage in the microscope maintained at the separation temperature $T_{0}$. This moment was designated as the beginning of the phase separation even though about 5 min were required to reach the thermal equilibrium at $T_{0}$. For the PS-DIDP system, experiments were carried out on two mixtures of 5.7 and $16.6 \mathrm{wt} \% \mathrm{PS}$ at various quench depths $\Delta T\left(\equiv T_{\mathrm{c}}(0)-T_{0}\right)$. All of the quenches are into the spinodal region. For the PS-PMPS system, the separation process was observed with a sample of $23.9 \mathrm{wt} \%$ PS at $\Delta T=5.4$ and $8.4^{\circ} \mathrm{C}$. The phase separation was observed about $1000 \mathrm{~min}$ for the PS-DIDP system and for 8000 min for the PS-PMPS system. Temperature fluctuations throughout the experiments in the former and the latter systems were within \pm 0.1 and $\pm 0.2^{\circ} \mathrm{C}$, respectively.

\section{RESULTS}

\section{PS-DIDP System}

Figure 1 shows the cloud and spinodal points for mixtures of PS and DIDP, in which the critical temperature and concentration are $48.5^{\circ} \mathrm{C}$ and 13 $\mathrm{wt} \% \mathrm{PS}$, respectively.

In Figure 2 are shown a series of typical photographs taken for a mixture of $16.6 \mathrm{wt} \% \mathrm{PS}$ at $\Delta T=$ $3.9^{\circ} \mathrm{C}$. At the beginning of the separation, there appeared a regular pattern of reticulate structure (Figure 2a), whose repeating unit, or characteristic 
length, developed as the separation proceeded (Figure 2b). This pattern took on the form of small droplets (Figure 2c), which grew larger with time

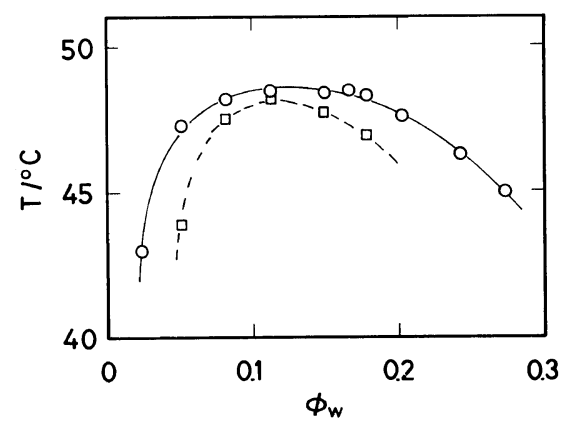

Figure 1. Cloud point curve $(O)$ and spinodal curve ( $\square$ ) for PS-DIDP system.

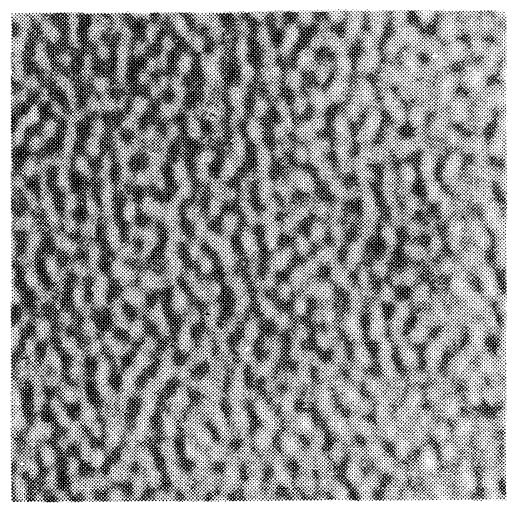

(a) $4.5 \mathrm{~min}$

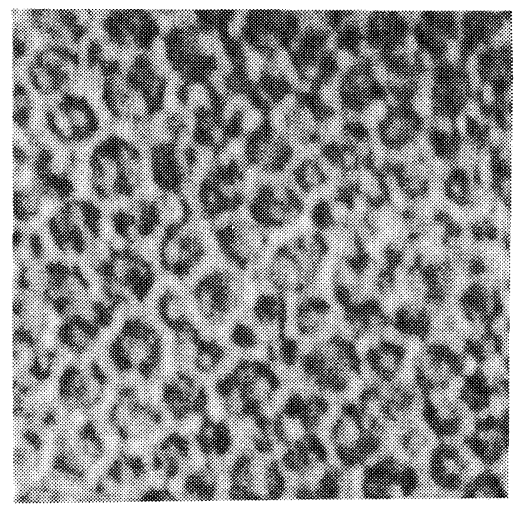

(c) $30.0 \mathrm{~min}$
(Figure 2d). Finally the movement of droplets occurred, which prevented further observation of the phase separation.

Figure 3 shows the time dependence of $\bar{d}$ for mixtures of 5.7 and $16.6 \mathrm{wt} \% \mathrm{PS}$ at various $\Delta T$. Droplet growth was rapid initially and then became slower for any $\Delta T$ and concentration $c$. At a fixed separation time $t$, droplet diameter increased with increasing $\Delta T$. As shown in Figure 4 , the time evolution of the average droplet number $\bar{N}$ per unit volume became faster as $\Delta T$ increased.

\section{PS-PMPS System}

The time evolution of the separated-phase structure for a blend of PS and PMPS was similar to that for the PS-DIDP mixture shown in Figure 2 except that the separation proceeded more slowly. Figure 5

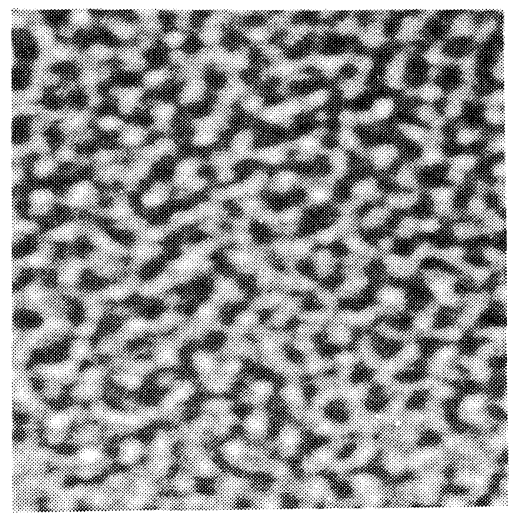

(b) $13.5 \mathrm{~min}$

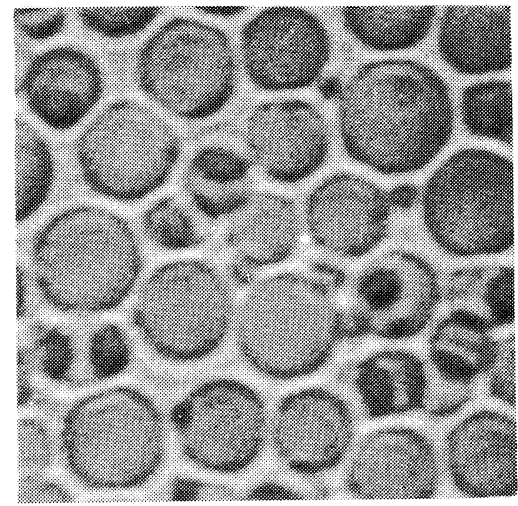

(d) $403 \mathrm{~min}$

\section{$\longleftrightarrow$}

Figure 2. Time variation in phase separation behavior for PS-DIDP system of $16.6 \mathrm{wt} \% \mathrm{PS}$ at $\Delta T=$ $3.9^{\circ} \mathrm{C}$. 


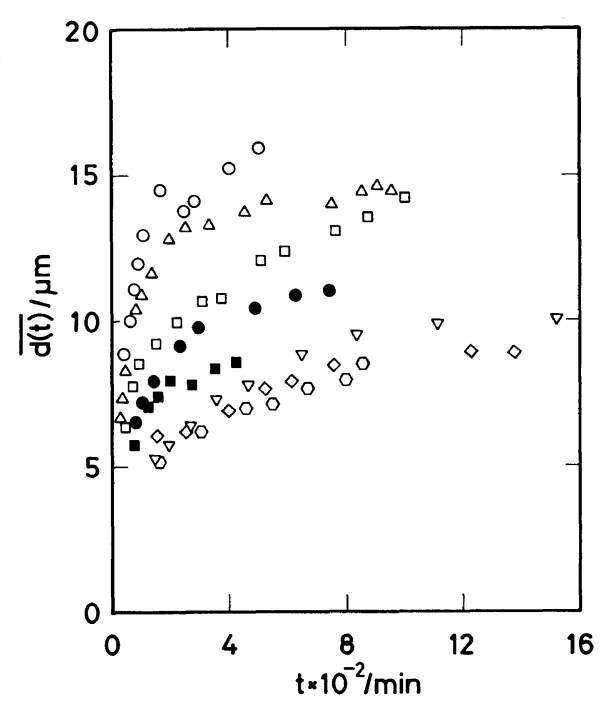

Figure 3. Time dependence of the average droplet diameter $\bar{d}$ for PS-DIDP system of $16.6 \mathrm{wt} \%$ PS at various quench depths; $(\bigcirc) 4.5^{\circ} \mathrm{C},(\triangle) 3.9^{\circ} \mathrm{C},(\square) 3.5^{\circ} \mathrm{C}$, $(\nabla) 2.5^{\circ} \mathrm{C}(\diamond) 1.8^{\circ} \mathrm{C},(\bigcirc) 1.1^{\circ} \mathrm{C}$, and of $5.7 \mathrm{wt} \% \mathrm{PS}$; (๑) $3.8^{\circ} \mathrm{C},(\square) 3.4^{\circ} \mathrm{C}$.

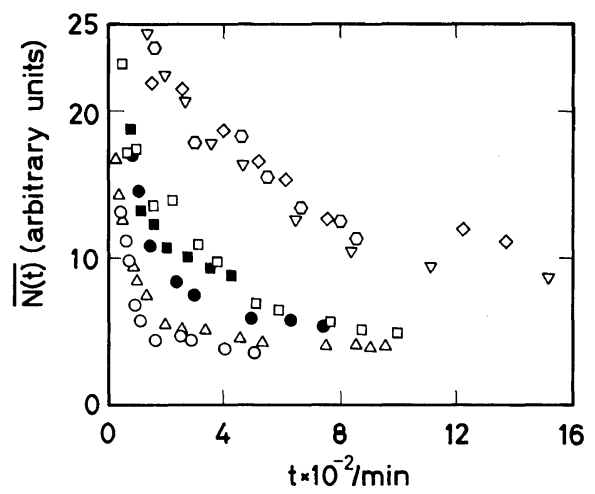

Figure 4. Time dependence of the average number of the droplet per unit volume $\bar{N}$ for PS-DIDP system. The symbols are the same as those in Figure 3.

shows the time dependence of $\bar{d}$ at $\Delta T=5.4$ and $8.4^{\circ} \mathrm{C}$. The three points for $\Delta T=5.4^{\circ} \mathrm{C}$ during a short period of time indicate the length of the repeating unit instead of the diameter, since we observed no droplet structure but a regular pattern of the reticulate structure like Figure $2 b$.

\section{DISCUSSION}

\section{PS-DIDP System}

The theories of the coalescence and OR mech-

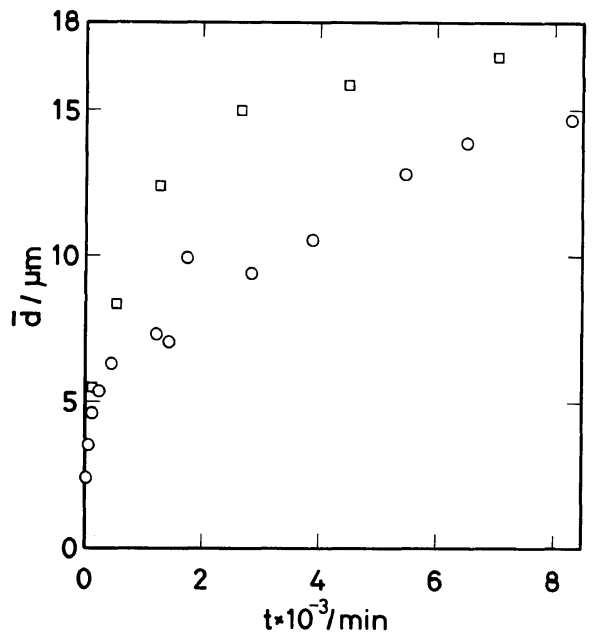

Figure 5. Time dependence of the average droplet diameter $\bar{d}$ for PS-PMPS system of $23.9 \mathrm{wt} \%$ PS at two quench depths; (O) $5.4^{\circ} \mathrm{C}$, ( $\square$ ) $8.4^{\circ} \mathrm{C}$.

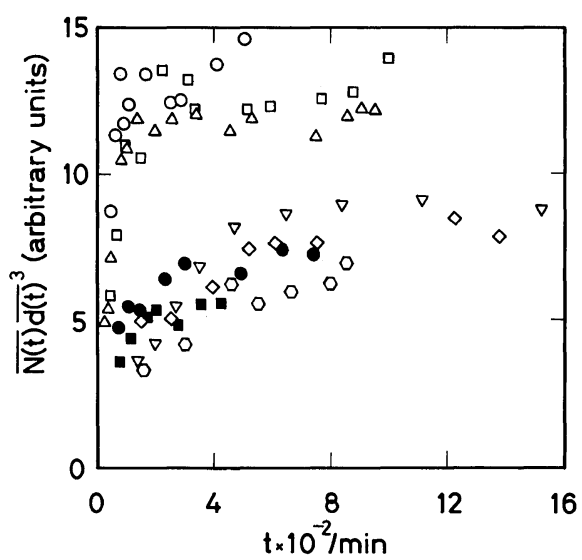

Figure 6. Time dependence of $\bar{N} \bar{d}^{3}$ for PS-DIDP system. The symbols are the same as those in Figure 3.

anisms assume that the total volume fraction of daughter phases does not change during the phase separation and is equal to the volume fraction of the minor phase $(\psi)$ macroscopically coexisting with the major phase following equilibrium. Therefore, the value of $\bar{N} \bar{d}^{3}$, which is proportional to the volume fraction of droplets, must be independent of the separation time $t$. In Figure 6, the values of $\bar{N} \bar{d}^{3}$ are plotted against $t$ for two mixtures of 5.7 and 16.6 $\mathrm{wt} \% \mathrm{PS}$ at various $\Delta T$. After a short period of rapid increase, these values level off to become nearly constant, though there is some scattering of the 


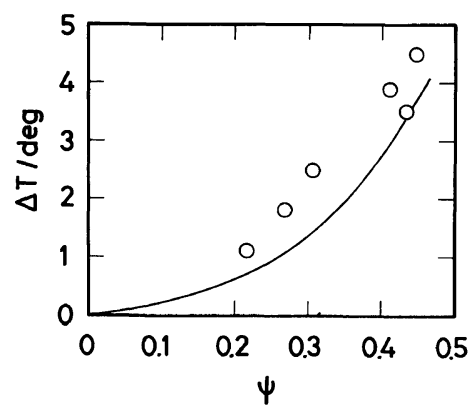

Figure 7. Quench depth dependence of the volume fraction of the droplet phase $\psi$ for PS-DIDP system. The solid curve was obtained from Figure 1 assuming the lever rule on the phase diagram.

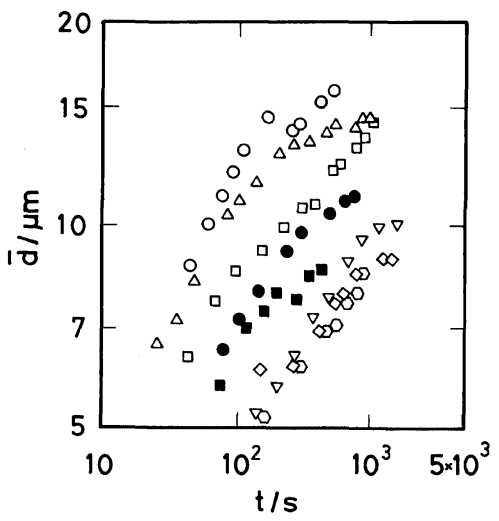

Figure 8. Time dependence of $\bar{d}$ for PS-DIDP system in log-log plot. The symbols are the same as those in Figure 3.

experimental data. In Figure 7, the volume fractions of the droplet phase $\psi$ calculated from the final values of $\bar{N} \bar{d}^{3}$ are shown as a function of $\Delta T$ for a mixture of $16.6 \mathrm{wt}^{\%} \%$ PS. The temperature dependence of $\psi$ obtained by microscopic measurements agrees with that calculated from the cloud point curve (Figure 1) by the lever rule (indicated by a solid curve). From this result, we conclude that the process observed was the coarsening process, such as coalescence and the Ostwald Ripening.

Figure 8 shows plots of $\log \bar{d} v s$. $\log t$, which give straight lines except at the end of the observation. Table I summarizes the values of $\phi$ in the powerlaw expression, $\bar{d} \propto t^{\phi}$ obtained from Figure 8 . The average value of $\phi$ is $0.30( \pm 0.09)$ in good agreement with the theoretical value $1 / 3$.

Generally, for a critical mixture, we can distinguish the coalescence mechanism from the OR
Table I. The exponent $\phi$ in the power-law relation, $\bar{d} \propto t^{\phi}$, for PS-DIDP system

\begin{tabular}{ccc}
\hline & $\Delta T$ & $\phi$ \\
\hline & 4.5 & 0.392 \\
& 3.9 & 0.354 \\
& 3.5 & 0.234 \\
& 2.5 & 0.339 \\
& 1.8 & 0.298 \\
$5.7 \mathrm{wt} \%$ & 1.1 & 0.279 \\
\hline & 3.8 & 0.305 \\
& 3.4 & 0.208 \\
\hline
\end{tabular}

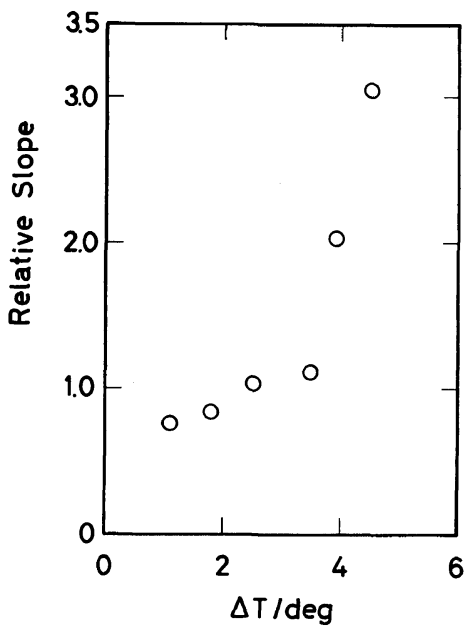

Figure 9. Relative slope in $\bar{d} v s . t^{1 / 3}$ against $\Delta T$ for PSDIDP system.

mechanism by the temperature dependence of the pre-factors of $t^{1 / 3}, k_{\mathrm{B}} T \psi / \eta$ and $\sigma M /\left(c_{\alpha}-c_{\beta}\right)^{2}$, in eq 1 and 2. In the former mechanism, the pre-factor should decrease gradually with increasing $\Delta T$ in the critical region, since the value of $\psi$ is approximately constant for a critical mixture and $\eta$ increases slowly with $\Delta T$ without singularity at the critical point $T_{\mathrm{c}}$. In the latter mechanism, the interfacial tension $\sigma$ must be dominant near $T_{\mathrm{c}}$ and yield a rapid increase in the magnitude of the pre-factor as the separation temperature departs from $T_{\mathrm{c}}$, since the values of $\sigma$ and $\left(c_{\alpha}-c_{\beta}\right)^{2}$ increase in accordance with the powerlaw against $\Delta T$ with $\mu=1.3$ and $2 \beta=0.7$, respectively. ${ }^{12}$ The pre-factors obtained from Figure 8 show rapid increase with increasing $\Delta T$ as illustrated in Figure 9. But in the present case, we can not distinguish between the mechanisms from Figure 
9, since the value of $\psi$ in eq 1 also increases with increasing $\Delta T$ (see Figure 7). The cross-over from the OR mechanism to the coalescence mechanism is theoretically predicted to occur at the small volume fraction $\psi$ of the daughter phase, such as at about $1 \%$ for binary mixtures of small molecules. ${ }^{1}$ This suggests that the coalescence mechanism holds for the present case in which the fraction $\psi$ exceeded $20 \%$ (see Figure 7 ).

For binary mixtures of $2-6$ lutidine and water, Goldburg et al. ${ }^{13}$ found the time dependence of $\bar{d}$ to obey the power-law $\bar{d} \propto t^{\phi}$ with $\phi=1 / 3$, but recently a more rapid growth of $\bar{d}$ with $\phi=1.7^{4}$ was found. This rapid growth was fully understood as resulting from the gravity effect which is not serious in polymer blends because of the high viscosity of the matrix phase. In fact, the present data, which show a rather slow growth at the end of the observation (see Figure 8), agree well with their early data. This fact suggests that there is no essential difference in the coarsening process in binary mixtures of small molecules and polymers.

\section{PS-PMPS System}

Figure 10 shows a plot of $\log \bar{d} v s . \log t$ for the blend of $23.9 \mathrm{wt} \% \mathrm{PS}$ at $\Delta T=5.4^{\circ} \mathrm{C}$, and that of

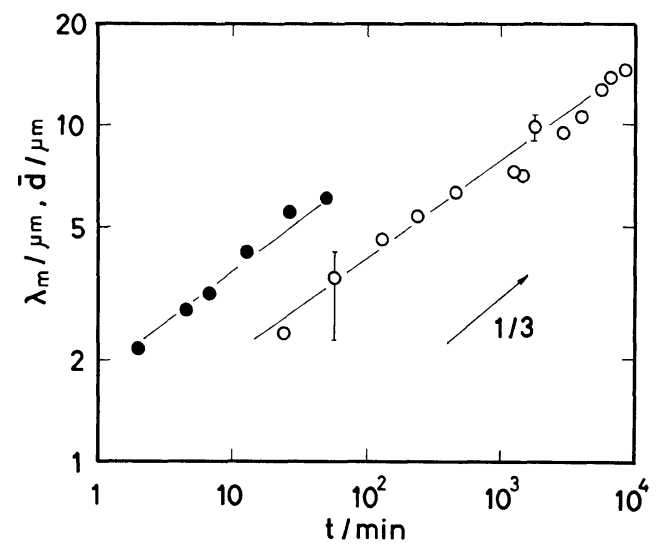

Figure 10. Time dependence of $\lambda_{\mathrm{m}}$ and $\bar{d}$ for PS-PMPS system of $23.9 \mathrm{wt} \%$ at $\Delta T=5.4^{\circ} \mathrm{C}: \bigcirc, \lambda_{\mathrm{m}}$ by light scattering; $\bigcirc, \bar{d}$ by microscope. $\log \lambda_{\mathrm{m}} v s . \log t$ for the same system obtained in a previous study by light scattering. ${ }^{8}$ Here, $\lambda_{\mathrm{m}}$ is the wave length at which the scattered intensity shows a maximum. The plot of $\log \bar{d} v s . \log t$ obeys the power-law $\bar{d} \propto t^{\phi}$ with $\phi=0.3$, as in the case of the PS-DIDP system, suggesting no essential difference in the coarsening process in the PS-DIDP and PSPMPS systems except for separation rates.

The change in $\lambda_{\mathrm{m}}$ with $t$ is also expressed by a power-law with $\phi=0.34$. The value of $\lambda_{\mathrm{m}}$ is twice as large as that of $\bar{d}$ in ranges of comparable time. If we difine a characteristic length $\bar{l}$ as $\bar{l}=\lambda_{\mathrm{m}}$ and $\bar{l}=2 \bar{d}$ for early and late stages, respectively, the time dependence of $\bar{l}$ is approximately represented by a single power-law $\bar{l} \propto t^{\phi}$ with $\phi=1 / 3$ throughout the phase separation including early and late stages. In a mixture of small molecules, 2-6 lutidine and water mixture, the rapid growth of droplets due to the gravity effect prevents a continuous increase in the characteristic length $\bar{l}$, but gives an inflection in the $\log \bar{l} v s . \log t$ curve in the cross-over region from the early to late stages. ${ }^{4}$

\section{REFERENCES}

1. E. D. Siggia, Phys. Rev., A20, 595 (1979).

2. I. M. Lifshitz and V. V. Slyozov, J. Phys. Chem. Solids, 19, 35 (1961).

3. C. Wagner, Zeit. Elektrochemie, 65, 581 (1961).

4. W. I. Goldburg, A. J. Schwartz, and M. W. Kim, Prog. Theor. Phys. Suppl., 64, 477 (1978).

5. J. J. van Aartsen and C. A. Smolders, Eur. Polym. J., 6, 1105 (1970).

6. L. P. McMaster, Adv. Chem. Ser., 142, 43 (1975).

7. G. G. A. Böhm, K. R. Lucas, and W. G. Mayes, Rubber Chem. Technol., 50, 714 (1977).

8. S. Nojima, K. Tsutsumi, and T. Nose, Polym. J., 14, 225 (1982).

9. S. Nojima and T. Nose, Polym. J., 14, 269 (1982).

10. K. Shinozaki, T. Hamada, and T. Nose, to be appeared in J. Chem. Phys.

11. N. Kuwahara, D. V. Fenby, M. Tamsky, and B. Chu, J. Chem. Phys., 55, 1140 (1971).

12. K. Shinozaki, T. V. Tan, Y. Saito, and T. Nose, to be appeared in Polymer.

13. W. I. Goldburg and J. S. Huang, "Fluctuations, Instabilities and Phase Transitions," T. Riste, Ed., Plenum Press, New York, N.Y., 1975, p 87. 